\begin{tabular}{|c|c|}
\hline IV Simpósio Paranaense de Modelagem, & Artigo: \\
\hline ISSN : 1984-7521 & Páginas: 89 - 96 \\
\hline
\end{tabular}

\title{
INFLUÊNCIA DA VAZÃO DE GÁS DISPERSANTE SOBRE A PERDA DE CARGA DE UM BICO ATOMIZADOR DO TIPO AIR- BLAST
}

\author{
Rafael Vieira Velozo, Jaqueline Catapan, Hortênsia Aparecida Busana, Rebeca Isolani Theis, \\ Leonardo Machado da Rosa, Jonathan Utzig, Waldir Pedro Martignoni, Henry França Meier* \\ Universidade de Blumenau-FURB,Blumenau-SC, meier@furb.br
}

\begin{abstract}
Bicos atomizadores são dispositivos que promovem a dispersão de carga líquida em diversas aplicações industriais. $O$ atomizador do tipo "air-blast”" funciona com o contato agressivo entre um gás dispersante e um líquido a ser disperso. A perda de carga é importante para o estudo de bicos atomizadores, pois afeta diretamente a efetividade da atomização e formação de gotículas. Dentre os fatores que influenciam a perda de carga de bicos, está a vazão de gás dispersante. O objetivo do estudo foi analisar o efeito da vazão de gás dispersante em relação à perda de carga do bico atomizador, e constatar qualitativamente a relação entre vazão de gás dispersante e de líquido dispersado. Os experimentos foram realizados por simulação numérica, e os resultados apresentaram que a perda de carga é diretamente proporcional à vazão de gás dispersante. Através dos contornos de fração volumétrica, foi possível avaliar qualitativamente a dispersão do líquido em função da vazão de gás. Palavras-chave: Spray, Atomização, Dispersor de carga, "Air-blast”, Perda de carga.
\end{abstract}

\section{Introdução}

Atomizadores são dispositivos presentes em diversas operações industriais. Eles são utilizados para auxiliar os processos através da dispersão do fluido, afim de que a etapa físico-química seja facilitada através do aumento da superfície de contato entre a carga atomizada e os reagentes. A atomização do tipo twin-fluid ocorre quando, dentro do bico do atomizador, acontece o contato entre uma corrente de fluido a ser atomizado e uma corrente de gás dispersante, sendo que a força cinética fornecida por esta última é suficiente para quebrar o jato contínuo de fluido em pequenas gotículas, chamadas de nuvem de spray.

A perda de pressão em bicos possui grande relevância para o estudo de atomização, pois é uma resposta muito sensível a alterações na configuração do equipamento, tanto quanto na escolha dos fluidos. Trabalhos da literatura constatam que parâmetros da geometria do bico tais como o diâmetro [1], o comprimento [2] e o ângulo de chanfro [2] alteram significativamente a perda de carga dentro do atomizador. Além disso, as propriedades do fluido atomizado também possuem efeito ativo nesta resposta, como foi constatado na literatura [4,5] a respeito da viscosidade. Ainda, estudos desenvolvidos [6] para analisar o efeito combinado de variáveis geométricas e condições de operação, como a vazão do fluido sobre a perda de carga do bico, permitiram a regressão de resultados experimentais em modelos para a predição da queda de pressão. Entretanto, estes estudos foram realizados em um bico de dispersão de sólidos, sendo então um escoamento gás-sólido.

Em vista disso, o objetivo do presente trabalho é avaliar a relação entre a velocidade do gás dispersante e o perfil de pressão dentro de um atomizador do tipo twin-fluid com configuração air-blast, sendo por sua vez um escoamento gás-líquido. As dimensões do problema físico foram suprimidas devido à confidencialidade.

\section{Modelagem Matemática}

O problema físico em estudo envolve dois fluidos de fases distintas em movimento e entrando em contato, ocorrendo com isto uma troca de energia e de

\section{4 e 15 de março de 2019 Curitiba - Paraná}


quantidade de movimento. Para isto, consideraram-se as equações de conservação para a modelagem do escoamento: a equação de conservação de massa, ou equação da continuidade, a equação de transporte de quantidade de movimento e a equação de conservação de energia.

A abordagem selecionada para realizar a modelagem multifásica foi a euleriana para as duas fases, onde a presença ou ausência de cada fase é determinada através da interpenetrabilidade das fases e da conservação de sua massa total. Devido à presença de interface bem definida, utilizou-se o método Volume of Fluid (VOF), capaz de modelar o breakup de correntes de líquido contínuo [7], entre outras aplicações.

A abordagem VOF consiste na modelagem multifásica de dois fluidos imiscíveis. Nas células que possuem duas fases, a fração volumétrica indica a presença da interface, que é transportada. Nestas posições, as propriedades da célula são mediadas em relação ao volume de cada fase. Por fim, as equações foram resolvidas em uma formulação transiente.

Deste modo, a equação da continuidade pode ser escrita em termos da fração volumétrica de uma das fases, como para a $q$-ésima fase da seguinte forma, apresentada pela Equação (1):

$\frac{1}{\rho_{\mathrm{q}}}\left[\frac{\partial}{\partial \mathrm{t}}\left(\alpha_{\mathrm{q}} \rho_{\mathrm{q}}\right)+\nabla \cdot\left(\alpha_{\mathrm{q}} \rho_{\mathrm{q}} \mathbf{v}_{\mathrm{q}}\right)=\mathrm{S}_{\alpha_{\mathrm{q}}}\right]$

onde $\alpha_{\mathrm{q}}$ é a fração volumétrica da $q$-ésima fase, $\rho_{\mathrm{q}}$ é a massa específica, e $\mathrm{S}_{\alpha_{\mathrm{q}}}$ representa um termo fonte. A equação possui como restrição que a soma das frações volumétricas das fases possua valor unitário. A fase gás é tratada como sendo um gás ideal, compressível, e a sua massa específica é estimada através de uma equação de estado. A fração volumétrica é resolvida utilizando uma formulação implícita.

Ainda, a equação de transporte de quantidade de movimento na abordagem VOF é resolvida apenas uma vez no domínio computacional, e o campo de velocidades resultante é compartilhado entre as fases envolvidas. A equação de transporte de quantidade de movimento então é dada pela Equação (2):

$\frac{\partial}{\partial \mathrm{t}}(\rho \mathbf{v})+\boldsymbol{\nabla} \cdot(\rho \mathbf{v v})=-\nabla \mathrm{p}+\boldsymbol{\nabla} \cdot\left[\mu\left(\nabla \mathbf{v}+\nabla \mathbf{v}^{\mathrm{T}}\right)\right]+\rho \mathbf{g}+\mathbf{F}$

onde o termo p é a pressão estática, $\rho \mathbf{g}$ é a força gravitacional e $\mathbf{F}$ são forças externas.

A conservação da energia também é calculada utilizando-se apenas uma equação para todas as fases envolvidas, conforme expresso na Equação (3):

$\frac{\partial}{\partial \mathrm{t}}(\rho \mathrm{E})+\boldsymbol{\nabla} \cdot(\mathbf{v}(\rho \mathrm{E}+\mathrm{p}))=\boldsymbol{\nabla} \cdot\left(\mathrm{k}_{\mathrm{eff}} \nabla \mathrm{T}-\sum_{\mathrm{j}} \mathrm{h}_{\mathrm{j}} \mathbf{J}_{\mathrm{j}}+\left(\tau_{\text {eff }} \cdot \mathbf{v}\right)\right)+\mathrm{S}_{\mathrm{h}}$

sendo $\mathrm{k}_{\mathrm{eff}}$ a condutividade efetiva envolvendo a condutividade turbulenta, dependente do modelo de turbulência, $\mathbf{J}_{\mathbf{j}}$ é o fluxo difusivo das espécies $\mathrm{j}, \tau_{\text {eff }}$ é o tensor tensão efetivo envolvendo a tensão turbulenta e $S_{h}$ é um termo fonte. A energia total (E) para a q-ésima fase é definida conforme a Equação (4):

$\mathrm{E}_{\mathrm{q}}=\mathrm{h}_{\mathrm{q}}-\frac{\mathrm{p}}{\rho_{\mathrm{q}}}+\frac{\mathrm{v}^{2}}{2}$

O modelo de turbulência escolhido para esta modelagem foi o Shear-Stress Transport (SST) k- $\omega$, que dos modelos de duas equações é o mais indicado para a problemática do presente estudo [8], pois apresenta acurácia tanto perto das paredes da geometria quanto nas regiões de livre escoamento. As equações de transporte para $k \mathrm{e}$ para $\omega$ são apresentadas respectivamente nas Equações (6) e (7): 
$\frac{\partial}{\partial \mathrm{t}}(\rho \mathrm{k})+\nabla \cdot(\rho \mathrm{kv})=\boldsymbol{\nabla} \cdot\left(\Gamma_{\mathrm{k}} \nabla \mathrm{k}\right)+\mathrm{G}_{\mathrm{k}}-\mathrm{Y}_{\mathrm{k}}+\mathrm{S}_{\mathrm{k}}$

$\frac{\partial}{\partial \mathrm{t}}(\rho \omega)+\boldsymbol{\nabla} \cdot(\rho \omega \mathbf{v})=\boldsymbol{\nabla} \cdot\left(\Gamma_{\omega} \nabla \omega\right)+\mathrm{G}_{\omega}-\mathrm{Y}_{\omega}+\mathrm{D}_{\omega}+\mathrm{S}_{\omega}$

onde os termos $G_{k}$ e $G_{\omega}$ representam a geração de energia cinética turbulenta e da taxa de dissipação específica, $\Gamma_{\mathrm{k}}$ e $\Gamma_{\omega}$ são a difusividade efetiva, $\mathrm{Y}_{\mathrm{k}}$ e $\mathrm{Y}_{\omega}$ são a dissipação devido à turbulência, $D_{\omega}$ representa o termo de difusão cruzada e $S_{k}$ e $S_{\omega}$ são termos fonte. Mais detalhes sobre o modelo de turbulência podem ser consultados na literatura [9].

\section{Experimentação Numérica}

A Figura 1 apresenta cortes do domínio de cálculo, nos quais é possível observar a injeção das fases. Nota-se que o líquido a ser atomizado é alimentado pelas laterais do bico, enquanto o gás dispersante é proveniente da parte inferior da geometria, entrando os dois fluidos em colisão na área denominada "região de contato". A linha utilizada para realizar a medição do perfil de pressão, localizada no centro do dispositivo, desde a entrada de gás até a saída do bico atomizador, encontra-se destacada em amarelo na Figura 1. Ao final dela, encontra-se a câmara de escoamento livre, onde o spray é formado e as gotículas são dispersas. As simulações numéricas foram executadas variando a vazão de gás dispersante no atomizador e medindo a pressão resultante.

O líquido a ser atomizado, a água, entra à temperatura e pressão atmosféricas de $25^{\circ} \mathrm{C}$ e $1 \mathrm{~atm}$, possuindo nestas condições massa específica de $998,2 \mathrm{~kg} / \mathrm{m}^{3} \mathrm{e}$ viscosidade absoluta de $10,03 \times 10^{-6} \mathrm{~Pa} \cdot \mathrm{s}$. O gás dispersante, ar atmosférico seco, também foi considerado à temperatura e pressão atmosféricas, sendo considerado compressível devido à proximidade em alguns casos da velocidade sônica, precisando então de uma equação de estado para a estimativa da massa específica, e sua viscosidade nessas condições é de $17,89 \times 10^{-6} \mathrm{~Pa} \cdot \mathrm{s}$

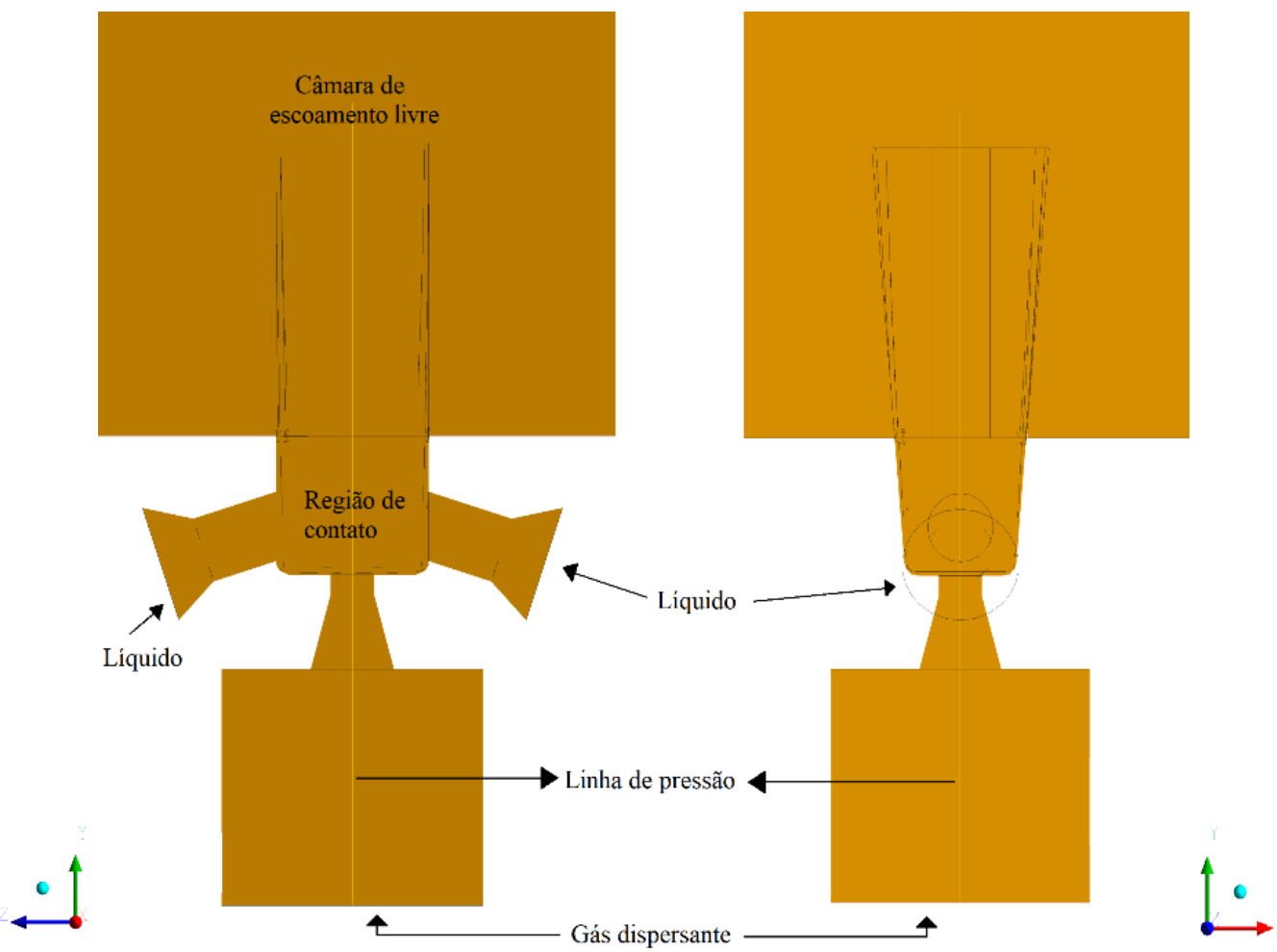

Figura 1 - Geometria do bico atomizador do tipo twin-fluid em disposição air-blast; vista em seções transversais nos planos (a) ZY e (b) XY. 
A malha numérica utilizada para o estudo possui cerca de 112 mil elementos, sendo ela mais refinada nas regiões de escoamento com maior velocidade e mais esparsa nas regiões com menor velocidade. Como pode-se observar na Figura 2, a região mais refinada possui elementos na ordem de $1 \times 10^{-6} \mathrm{~m}$, enquanto que a mais esparsa possui células na ordem de $1 \times 10^{-5} \mathrm{~m}$. O menor volume de controle possui aproximadamente $1 \times 10^{-10} \mathrm{~m}^{3}$, e o maior volume na ordem de $1 \times 10^{-6} \mathrm{~m}^{3}$. O passo de tempo com número de Courant satisfatório foi o de $1 \times 10^{-5} \mathrm{~s}$ e todos os casos foram resolvidos entre 0,190 e 0,025 s de tempo real, dependendo da vazão do gás dispersante, o que se mostrou ser tempo o suficiente para o escoamento ser completamente desenvolvido no interior do bico.

O tempo de duração das simulações foi de aproximadamente 10 dias, cada caso utilizando 5 processadores, com pequenos intervalos entre este período para coleta de resultados e verificação de contornos de fração volumétrica afim de analisar o desenvolvimento do escoamento. As simulações foram conduzidas utilizando-se do código comercial ANSYS ${ }^{\circledR}$ Fluent versão 19.0.

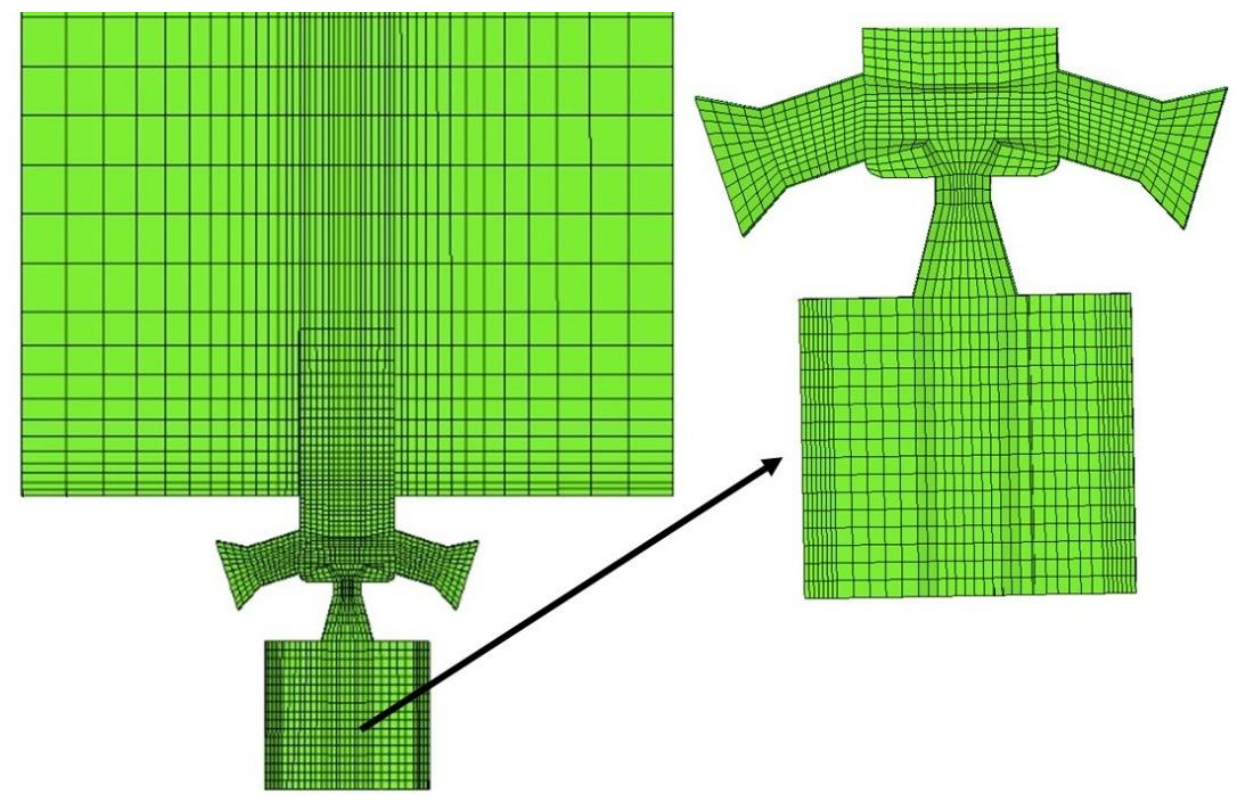

Figura 2 - Malha numérica utilizada para a experimentação numérica (a) na região próxima ao bico injetor, e (b) detalhe na alimentação dos fluidos.

As condições de contorno dos experimentos numéricos incluem a vazão de líquido dispersado de $1500 \mathrm{~kg} / \mathrm{h}$, sendo este água à pressão e temperatura atmosférica, e a condição de saída foi uma configuração de pressão ambiente. A vazão de gás dispersante foi simulada com um valor diferente para casa caso, iguais a 5, 15, 25, $35 \mathrm{e}$ $45 \mathrm{~kg} / \mathrm{h}$ de ar atmosférico. Resultados obtidos para fração volumétrica, velocidade e pressão foram avaliados.

\section{Resultados e Discussão}

O campo de pressão e vetores de velocidade são apresentados nas Figuras 3 e 4, respectivamente. Pode-se observar que a maior queda de pressão ocorre na região de afunilamento do duto de gás dispersante, logo antes do mesmo entrar na região de contato com o líquido atomizado; além disso, ocorrem regiões de pressão relativa negativa nesta mesma região de contato. 


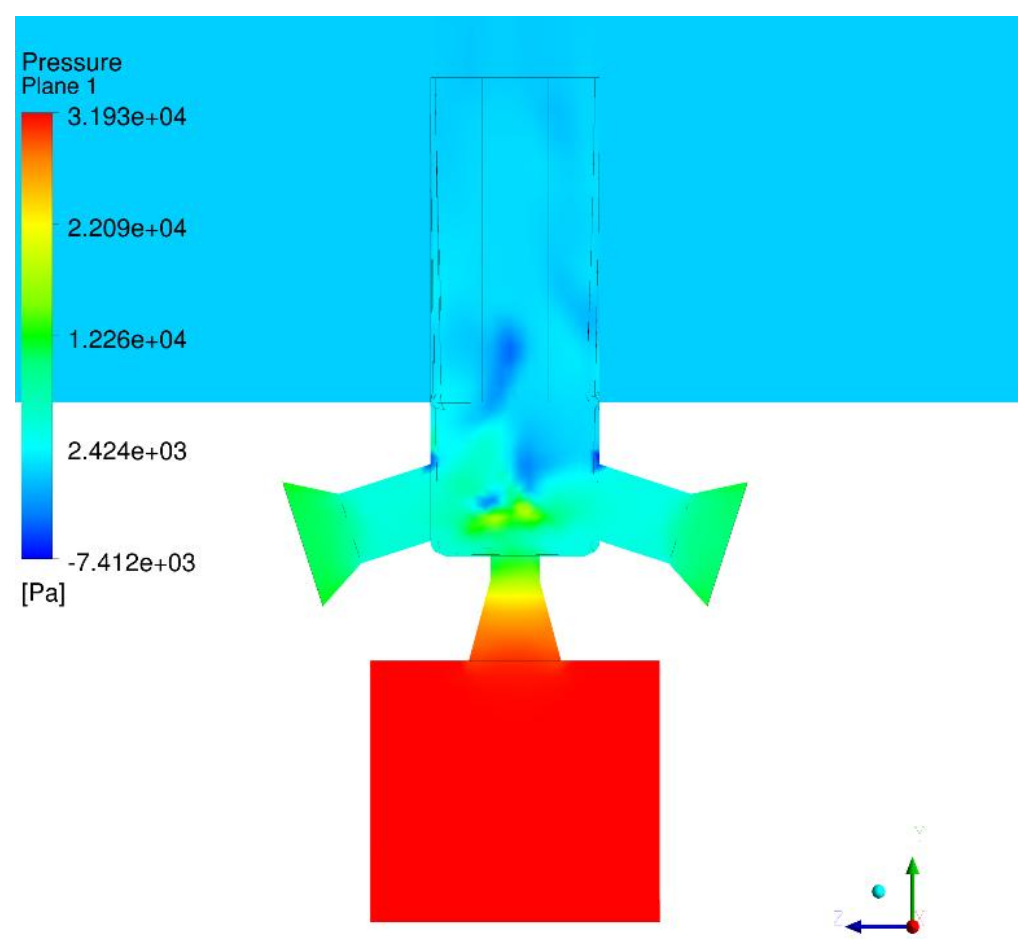

Figura 3 - Campo de pressão para vazão de $25 \mathrm{~kg} / \mathrm{h}$ de gás dispersante.

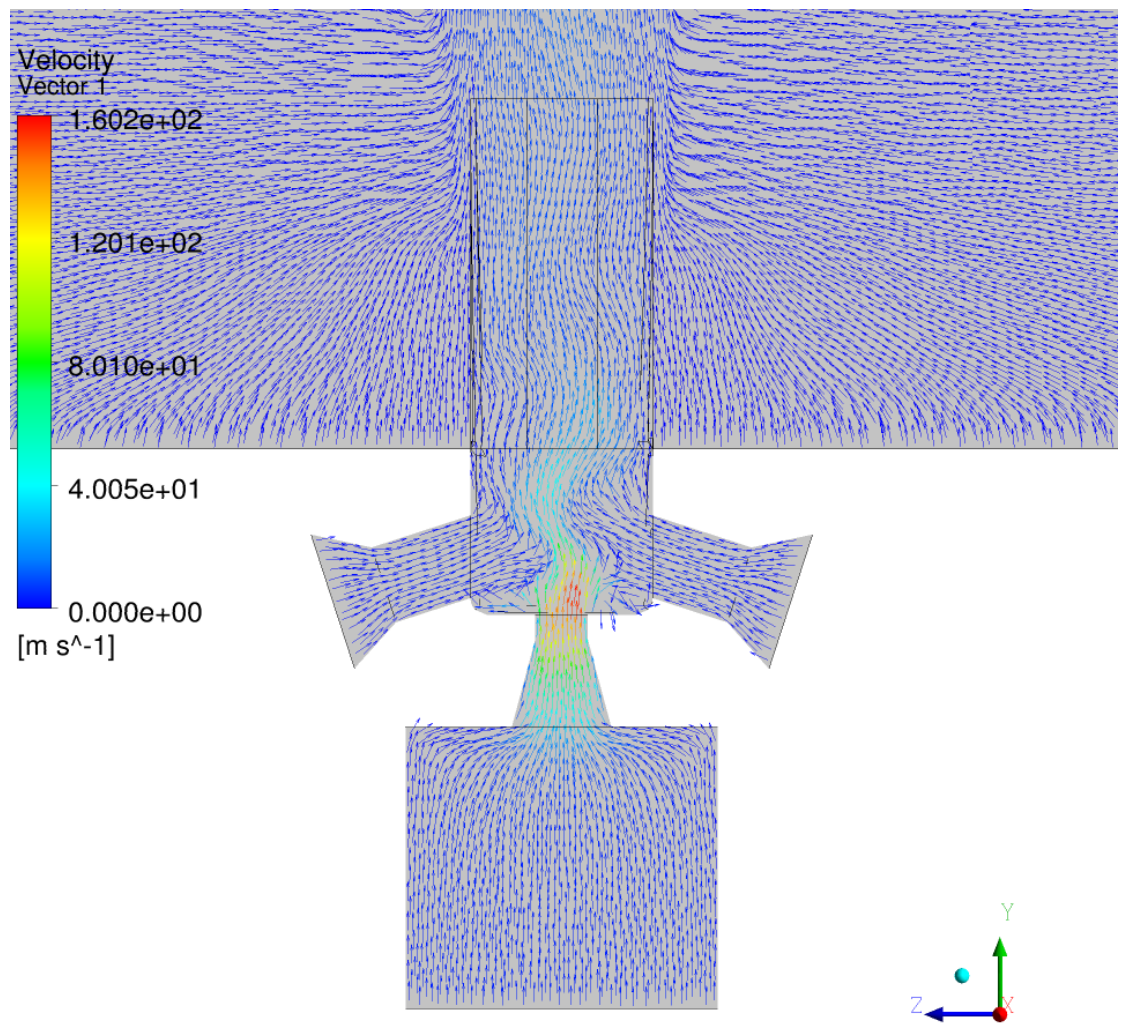

Figura 4 - Vetores de velocidade para vazão de $25 \mathrm{~kg} / \mathrm{h}$ de gás dispersante.

Através dos vetores, pode-se notar que as maiores velocidades se concentram na região de saída do gás dispersante, chegando a aproximadamente $160 \mathrm{~m} / \mathrm{s}$ para a vazão de $25 \mathrm{~kg} / \mathrm{h}$. Nesta condição, atinge-se um valor de 0,47 para o número de Mach. No caso com $45 \mathrm{~kg} / \mathrm{h}$ de gás dispersante, chegou-se próximo à condição de escoamento sônico, com número de Mach igual a 0,94. 


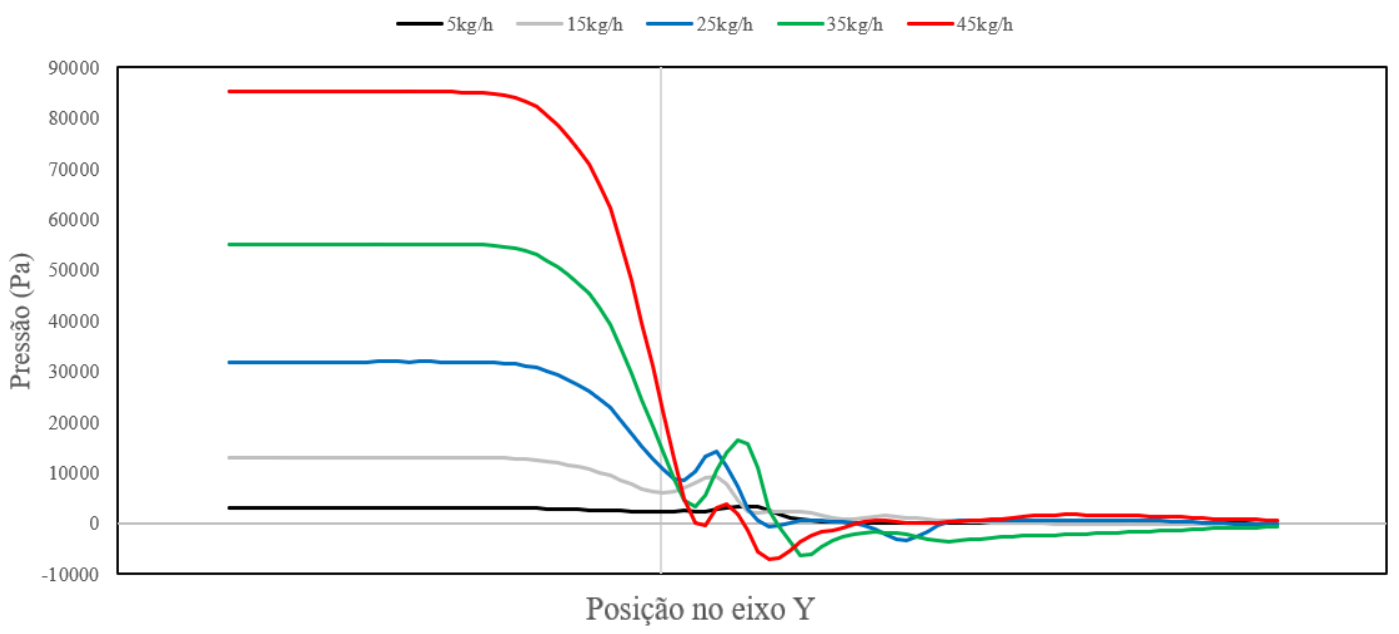

Figura 5 - Perfis de pressão medido na linha ao centro da geometria do atomizador para diferentes vazões de gás dispersante.

Na Figura 5 é possível observar que a pressão é praticamente constante antes da entrada de gás dispersante. Na entrada do bico, onde há o afunilamento do duto, a pressão sofre uma queda drástica, seguida por oscilações devido à região de contato com o líquido, até por fim estabilizar na pressão atmosférica. Também é possível notar que quanto maior a vazão, maior é a queda de pressão.

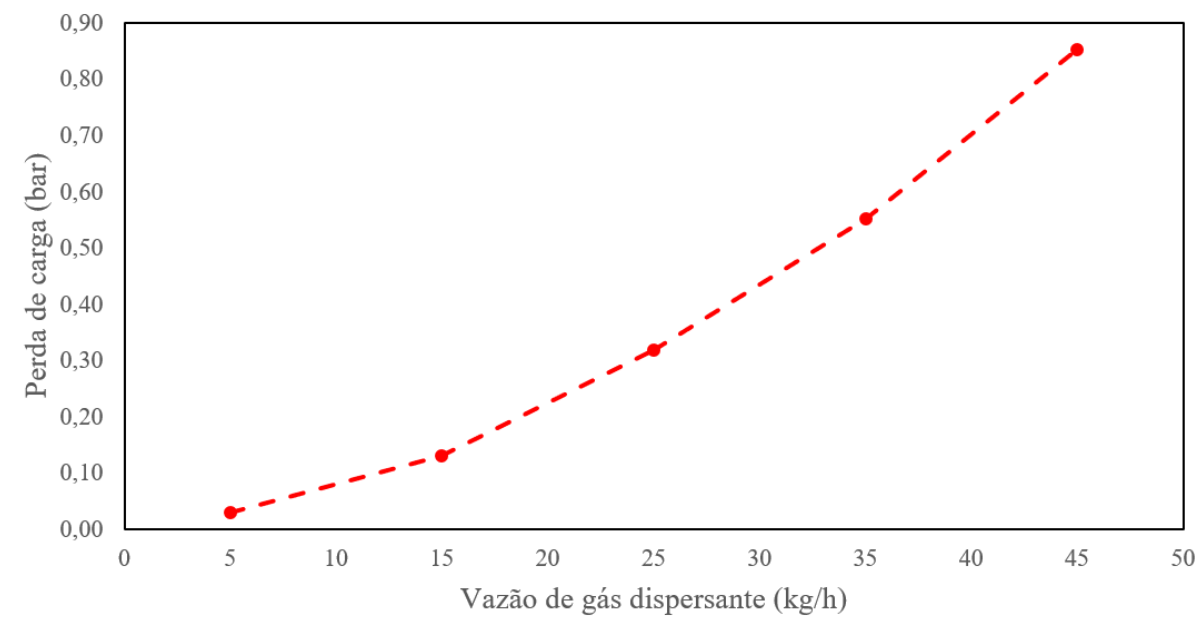

Figura 6 - Relação entre vazão de gás dispersante e perda de carga no bico atomizador.

$\mathrm{Na}$ Figura 6, apresenta-se o comportamento da perda de carga dentro do bico atomizador. Nota-se que a perda de carga eleva-se em função da vazão de gás dispersante. Ainda, a maior perda de carga, de aproximadamente 0,9 bar, foi resultada pela maior vazão de gás dispersante, de $45 \mathrm{~kg} / \mathrm{h}$.

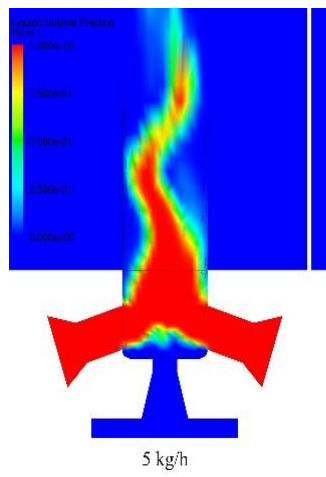

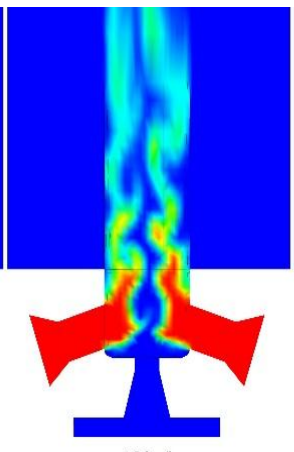

$15 \mathrm{~kg} / \mathrm{h}$

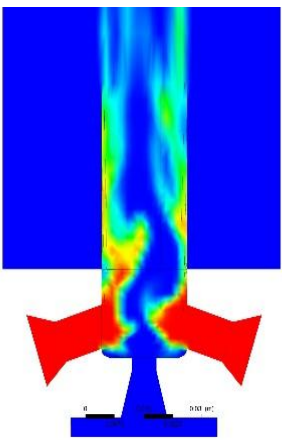

$25 \mathrm{~kg} / \mathrm{h}$

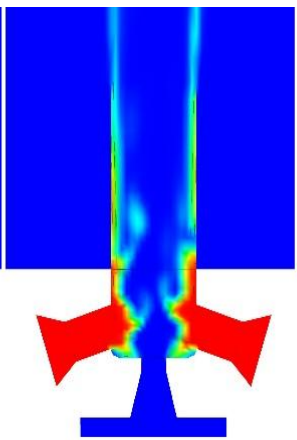

$35 \mathrm{~kg} / \mathrm{h}$

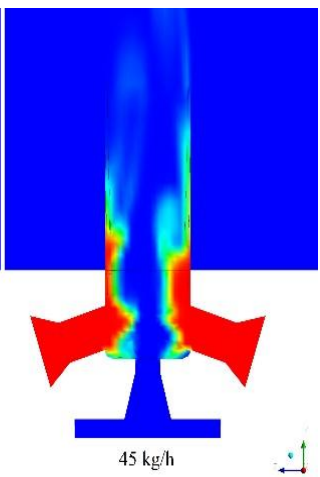

$45 \mathrm{~kg} /$ $\therefore$

Figura 7 - Contornos instantâneos de fração volumétrica de líquido disperso para as vazões de (a) $5 \mathrm{~kg} / \mathrm{h}$, (b) $15 \mathrm{~kg} / \mathrm{h}$, (c) $25 \mathrm{~kg} / \mathrm{h}$, (d) $35 \mathrm{~kg} / \mathrm{h}$ e (e) $45 \mathrm{~kg} / \mathrm{h}$. 
Na Figura 7 é possível observar o efeito da vazão de gás dispersante sobre a distribuição de líquido. Nas vazões intermediárias de 25 e $35 \mathrm{~kg} / \mathrm{h}$, o líquido deixa a câmara de escoamento livre em uma nuvem menos concentrada de líquido, indicando gotículas. Nas primeiras vazões, a velocidade do gás dispersante não é suficiente para quebrar o jato de líquido em gotículas, enquanto que na última vazão a velocidade é tão alta que o jato de líquido é diretamente arremessado para as paredes, evitando a formação de um spray uniforme na saída do bico.

\section{Conclusão}

Resultados obtidos através de simulações numéricas demonstraram-se coerentes fisicamente, sendo dentro do esperado para bicos atomizadores. Os valores de perda de carga obtidos poderão ser utilizados em estudos mais detalhados sobre bicos atomizadores do tipo air-blast, através da linha de tendência gerada com os experimentos numéricos. Também foi possível observar que o escoamento logo na saída de gás no bico possui comportamento quase sônico nas vazões mais elevadas, podendo chegar a sônico, como é o caso de alguns atomizadores industriais. Resultados obtidos em simulações também demonstraram o efeito da vazão de gás dispersante sobre a distribuição de líquido, o que permite avaliar a qualidade do spray em diferentes condições operacionais.

\section{Agradecimentos}

Este estudo foi financiado com o apoio da Petróleo Brasileiro S.A. (Petrobras), através do termo de cooperação $\mathrm{n}^{\circ} 5850.0107354 .18 .9$.

\section{Referências bibliográficas}

1. A. Royne, C.J. Dey, "Effect of nozzle geometry on pressure drop and heat transfer in submerged jet arrays", International Journal of Heat and Mass Transfer 49 (2006) 800-804

2. Y.F. Tuo, L.H. Yang, T. Guo, L.J. Zhang, "A calculative formula and experimental study on loss-of-head of taper nozzle", Water Saving Irrigation 4 (2005) $14-16$

3. L.A. Brignoni, S.V. Garimella, "Effects of nozzle-inlet chamfering on pressure drop and heat transfer in confined air jet impingement", International Journal of Heat and Mass Transfer 43 (2000) 1133-1139

4. C. James, M.Ware, J. Grant, A. Thorpe, A. Michael, F. Senftle, S. Smith, "Flow equation for an ideal or viscous gas through a nozzle: application to cascade impactors", Experimental Thermal and Fluid Science 25 (2001) 421-427

5. D.A. McNeil, A.D. Stuart, "Vertically upward two-phase flow with a highly viscous liquid-phase in a nozzle and orifice plate", International Journal of Heat and Fluid Flow 25 (2004) 58-73

6. W. Huang, X. Gong, X. Guo, Z. Dai, H. Liu, Z. Cao, C. Wang. "Study of the pressure drop of dense phase gas-solid flow through nozzle". Powder Technology, 2009, 189(1), 82-86.

7. C. W. Hirt, B. D. Nichols. "Volume of fluid (VOF) method for the dynamics of free boundaries". Journal of Computational Physics. 1981. 39 (1): 201-225

8. J. Hasan, A. Sepideh, X. Jie, D. C. Xiao, S. Cordelia, W. W. Meng. "On the effect of turbulence models on CFD simulations of a counter-current spray drying process". Chemical Engineering Research and Design, 2019. (141):592-607.

9. F. R. Menter. "Two-Equation Eddy-Viscosity Turbulence Models for Engineering Applications". AIAA Journal. 1994. 32(8). 1598-1605 
